\title{
Podoplanin as Key Player of Tumor Progression and Lymph Vessel Proliferation in Ovarian Cancer
}

\author{
IONUT MARCEL COBEC $^{1,2}$, IOAN SAS $^{1}$, LAURENȚIU PIRTEA ${ }^{1}$, ANCA MARIA CIMPEAN ${ }^{3}$, \\ AURICA ELISABETA MOATAR ${ }^{2}$, RALUCA AMALIA CEAUS, ${ }^{3}$ and MARIUS RAICA ${ }^{3}$ \\ ${ }^{1}$ Department of Obstetrics and Gynecology, and ${ }^{3}$ Department of Microscopic Morphology/Histology, \\ Angiogenesis Research Center, Victor Babes University of Medicine and Pharmacy, Timisoara, Romania; \\ ${ }^{2}$ Department of Obstetrics and Gynecology, Diakonie Klinikum, Schwäbisch Hall, \\ Academic Hospital of the Heidelberg University, Heidelberg, Germany
}

\begin{abstract}
Background/Aim: Podoplanin plays a key role in tumor progression and metastasis. We evaluated lymphatics proliferation rate and podoplanin expression in tumor cells of ovarian carcinoma. Materials and Methods: Seventy-five paraffin-embedded specimens of ovarian cancer were immunohistochemically assessed in order to quantify peritumoral (LMVDP) and intratumoral (LMVDT) lymphatic microvessel density of proliferating lymphatics and for podoplanin variability in tumor cells. Results and Conclusion: LMVDT correlated with proliferating tumor vessels located in the peritumoral area $(p=0.024)$ and with the number of mature vessels located in the intratumoral area $(p<0.0001)$, while LMVDP correlated with peritumoral mature vessels $(p<0.000 l)$. Proliferating tumor cells at the invasive front were highly positive for podoplanin. To the best of our knowledge, this study represents the first assessment of lymphatic endothelial cell proliferation correlated with podoplanin expression in tumor cells from ovarian cancer. Our data support podoplanin as a potential target that may help reduce ovarian cancer dissemination and lymphatic metastasis.
\end{abstract}

Podoplanin (D2-40), is a specific marker for lymphatic endothelial cells since it is not expressed in endothelial cells from blood vessels, with the exception of high endothelial venules located inside lymph node parenchyma (1-3).

Correspondence to: Anca Maria Cimpean, MD, Ph.D., Professor of Histology, Department of Microscopic Morphology/Histology, Angiogenesis Research Center, Piata Eftimie Murgu 2, 300041 Timisoara, Romania. Tel: +40 720060955, e-mail: ancacimpean1972@ yahoo.com

Key Words: Podoplanin, lymphatic endothelial cells, ovarian cancer, lymphatic metastasis.
Nevertheless, podoplanin is not restricted to the endothelial level, its expression also being evidenced in basal cells of the stratified epithelium that lines the oral cavity (4) and basal epithelial cells of the uterine cervix $(5,6)$. At the level of the stromal compartment, podoplanin expression is not only increased in myofibroblast-like cells type, its weak expression in normal connective tissue being well known, but also in pathological situations such as Dupuytren disease or in tumor-associated stroma (7). In the normal ovary, podoplanin expression has been less characterized, not only concerning its endothelial expression, but also its expression in the ovarian stroma. The ovarian stroma is known to have specific spindle-like cells. The role and involvement of podoplanin in ovarian physiology is controversial and incompletely characterized at the time being. Based on these data, one of the aims of our study was the detailed description of podoplanin expression and distribution in the normal ovary adjacent to ovarian tumor.

Podoplanin is a well-known key player involved in tumor progression and metastasis. Various tumor types, such as breast or gastric cancer and different sarcomas, are characterized by an increased expression of podoplanin in tumor cells, especially in cells located at the tumor periphery and in between the tumor stroma and the tumor mass. The podoplanin antibody developed by Kato and Kanako, which possesses targeted action towards this marker, is a proof of the importance of podoplanin expression in tumor cells. At the time being, this antibody is in advanced testing (8).

Lymphatic microvessel density (LMVD) in ovarian tumors was exclusively quantified with the use of D2-40 $(9,10)$, the lymphatic endothelial cell proliferative index was not taken into consideration. Another aspect with indirect consequences on the evaluation of podoplanin in ovarian tumors is represented by the global evaluation of LMVD. In the previously mentioned studies, the global LMVD was assessed but the differences between intratumoral and 
peritumoral areas regarding lymphatic vessel density (LMVDT and LMVDP, respectively) were not consistently evaluated, nor was the LMVD for normal ovarian tissue adjacent to tumor tissue. Podoplanin expression in tumor ovarian cells was reported by several researchers, although a well-defined prognostic and therapeutic impact is not available at the moment $(11,12)$. Lymphatic vessel morphology in ovarian tumors has been scarcely studied.

Based on the above described information, we aimed to evaluate tumor lymphangiogenesis in malignant or borderline ovarian lesions through examining the LMVD correlated with the endothelial cell proliferation index. Additionally, we evaluated correlation of podoplanin expression with the proliferation index in tumor cells as a useful tool for a better understanding of the lymphangiogenesis mechanism.

\section{Materials and Methods}

Seventy-five archival paraffin-embedded specimens of ovarian cancer were selected. Two experienced histopathologists reviewed the cases and selected those specimens appropriate for immunohistochemistry. Three-micrometer serial sections were obtained from each case. As a proper method for co-localization of lymphatic endothelial marker (podoplanin, clone D2-40) and proliferation marker (Ki67, clone MIB1) we selected a doublestaining method able to be performed in a fully automated manner by using BOND MAX autostainer (Leica Microsystems, Newcastle, UK) and a compatible standardized kit Bond Polymer Refine Detection System Brown for visualization in brown of Ki67 antigen (Leica Microsystems) followed by Bond Polymer Refine Red Detection System to highlight expression of podoplanin in red inside lymphatic endothelial cells (Leica Microsystems). All double staining steps were strictly controlled using a standardized protocol provided by the manufacturer. Immunohistochemical specimens were semi-quntitatively evaluated regarding LMVDP and LMVDT of proliferating lymphatics (using the 'hot-spot' method) and also for the expression of podoplanin in tumor cells, knowing that podoplanin is currently considered as a factor favoring tumor progression and metastasis. LMVDP and LMVDT were then correlated with tumor grade and tumor type.

Statistical analysis was performed by using SPSS 19 software (IBM Corp., Armonk, NY, USA). Image acquisition and processing were performed by using AxioCam 506 Color Research Microscope (Zeiss, Jena, Germany).

\section{Results}

In normal ovarian tissue adjacent to the tumor, we observed an intense podoplanin expression of the covering epithelium of the ovary, of the lymphatic vessels, and also in the spindle-like areas of the ovarian stroma. The cuboidal cells that form the ovarian covering epithelium were positive for podoplanin at their apical pole and along their lateral microdomains. The ovarian stroma located immediately beneath the covering epithelium presented weak and inconstant expression for podoplanin, except for some smallcaliber lymphatic vessels. These vessels presented a patent lumen and the lining endothelial cells exhibited constant expression of podoplanin. In the medulla, podoplanin expression had increased intensity. Its expression was identified in the spindle-like stromal cells that presented a storiform distribution.

At the same time, several macrophage-like cells were identified as cell clusters crowded around the albicans body. Podoplanin was absent from the albicans bodies with the exception of rare fibrocyte-like cells. An aspect that should not be neglected is the crowding of D2-40-positive macrophage-like cells, which were distributed in large groups around the albicans bodies. A particular aspect that we noticed was that in some cases, around the albicans body, D2-40-positive lymphatic vessels were present with an increased density compared to those found in the ovarian stroma from other areas. D2-40-positive lymphatic vessels were rarely encountered inside the albicans body. The lymphatic vessels exhibited a specific morphology with a sinuous aspect or presenting intraluminal pillars, suggesting the existence of active lymphangiogenesis in those areas. Some lymphatic vessels around the albicans bodies had the tendency to invade them, although no lymphatic endothelial proliferation was observed. In the areas where lymphatic vascular density (20-40 vessels/field at $\times 200$ in the ovarian cortex) was higher, fibroblast-like cells were negative for podoplanin. In the ovarian medulla, the density of D2-40 positive macrophages was much higher than that evidenced by the cortex. In ovarian medulla, lymphatic vessel density was significantly increased compared with that observed in the ovarian cortex. The expression of D2-40 in this area was restricted to the lymphatic endothelium. Large blood vessels, both arterial and venous, were negative for D2-40. D2-40 expression in the tumor tissue was evaluated at the lymphatic vessel endothelium. Its expression was also observed in tumor cells and appeared to differ according to the different histopathological types of ovarian cancer.

Our results regarding the evaluation of tumor lymphatic vessels included the differential quantification of the LMVD in tumoral and peritumoral areas. This differential quantification was particularly made through the addition of the proliferative grade which was analyzed by means of D240/Ki67 double immunostaining.

LMVDP was found to be between 0 and 40 vessels/field $\times 200$. Lymphatic vessels were grouped around the tumor areas and presented the tendency to invade them. Although these lymphatic vessels were distributed between normal and tumor ovarian tissue, their morphology was significantly different in comparison to lymphatic vessels from normal ovary. The morphological heterogeneity was defined through the presence of large-caliber lymphatic vessels, with an irregular lumen, often exhibiting a discontinuous wall and with massive tumor cell invasion, sometimes with a high proliferative grade. Speficif morphological features were 
present suggesting an extremly active peritumoral lymphangiogenesis. This aspect is supported by two microscopically active elements: the presence of cytoplasmic expansions of the endothelial cells towards the lumen (Figure 1a, thin arrows) as the first step of intussusception, followed by the highlighting of the well-outlined pillars which completely split the vessel (Figure 1a, yellow arrow). This second aspect resulted in the existence of two distinct lymphatic vessels, initially not invaded and afterwards exhibiting massive tumor cell invasion. Intussusception was accompanied by lymphatic endothelial cell proliferation, this combined aspect very often being encountered in the peritumoral areas of malignant ovarian tumors (Figure 1b). It seems that the intussusception phenomenon was concomitant with tumor invasion. Most peritumoral lymphatic vessels presented intraluminal pillars, and massive tumor emboli, which comprised of proliferating tumor cells that sometimes dilacerated the intraluminal pillars. This aspect was probably the main mechanism of massive dissemination of ovarian tumor cells. A feature that should be taken into consideration is that podoplanin was intensely expressed in nucleated blood elements identified not only inside the large peritumoral blood vessels but also in the plasma.

Intratumoral lymphatic vessels also presented intense heterogeneity, not only at the morphological level but also regarding the LMVD and endothelial cell proliferation. Intratumoral vessels with visible lumen were observed at the periphery of the tumor areas. Inside tumor, we observed two lymphatic vascular patterns: lymphatic vessels with collapsed lumen and lymphatic vessels with a visible lumen lined by D2-40-positive endothelial cells. In some ovarian tumors, LMVDT quantification was difficult to estimate due to the fact that the tumor cells either focally expressed podoplanin or its expression was homogenously distributed. However, these tumoral areas were characterized by the presence of lymphatic vessels, but we observed a tendency for D2-40-positive tumor cells to define vascular-like spaces similar to vascular mimicking, recognized as a mechanism of angiogenesis in some tumor types such as melanomas and gliomas. Endothelial proliferation was present, but we observed discontinuities in the wall of the intratumoral lymphatic vessels, a feature that may be due to massive intravascular invasion in the intratumoral area.

The number of the intraluminal pillars was increased in the areas where we observed several macrophages surrounding the peritumoral lymphatic vessels. The macrophages located in the peritumoral areas were intensely positive for D2-40. D2-40-positive macrophages were not specific to the peritumoral area, they were also encountered in the intratumoral areas, especially in cases where the tumor cells were D2-40-negative. The LMVDT ranged between 0 and 17 vessels/field at $\times 200$, the highest frequency being consistently seen in serous and mucinous papillary cystadenocarcinoma cases, the majority being graded as G3. Regarding their morphology, the intratumoral vessels were of small caliber, with a rarely visible lumen, most probably collapsed due to intratumoral pressure given by rapid tumor cell proliferation (Figure 2).

The average LMVDT of the intratumor vessels was three times lower than that estimated in the case of peritumoral lymphatic vessels (3.33 versus 9.02). The intratumoral lymphatic vessels exhibited a low rate of endothelial cell proliferation compared to the peritumoral lymphatic vessels. Approximately $50 \%$ of the cases presented proliferating vessels in the tumor areas. Very often the proliferating vessels exhibited a visible lumen and intravascular tumor emboli were present.

Statistical analysis of the total and proliferating lymphatic vessels showed that LMVDT was statistically significantly correlated with the number of proliferating tumor vessels located in the peritumoral area $(p=0.024)$ and with the number of mature vessels located in the intratumoral area $(p<0.0001)$. The LMVDPT was also statistically significantly correlated with the value estimated for peritumoral mature vessels $(p<0.0001)$. Statistical data are detailed in Table I.

The analysis of the influence of tumor grading on the proliferative index of lymphatic endothelial cells and on the LMVDT and LMVDP was considered a useful parameter in our study (Table II). Podoplanin expression in the tumor cells of ovarian carcinomas represents a challenge for future targeted therapies against metastasis and tumor growth. The expression of podoplanin in the tumor cells of ovarian carcinomas appears to be heterogeneous within the same tumor. At the same time, its expression varies from one tumor to another, depending on tumor type. Moreover, the proliferative grade of the tumor cells seems to influence podoplanin expression. Tumor cells with anaplastic morphology exhibited an increased intensity of podoplanin expression (Figure 3a). Podoplanin was consistently intensely expressed in tumor cells from the proliferative front, situated between the tumor stroma and the tumor mass (Figure 3b). Tumors that presented a papillary aspect were consistently positive, but heterogeneous, for podoplanin. Two patterns of expression were observed, namely: a cytoplasmatic pattern and an apically restricted one, which was noted in the epithelial cells that covered the connective axes in the carcinomas presenting a papillary aspect. The proliferative grade of the tumor cells was not influenced by the presence of podoplanin in tumor cells.

\section{Discussion}

Ovarian cancer remains a major health issue in the female population, not only in Romania but also worldwide. Ovarian cancer is very often diagnosed in the peritoneal metastasis stage, when chemotherapy and radiotherapy are less efficient. 

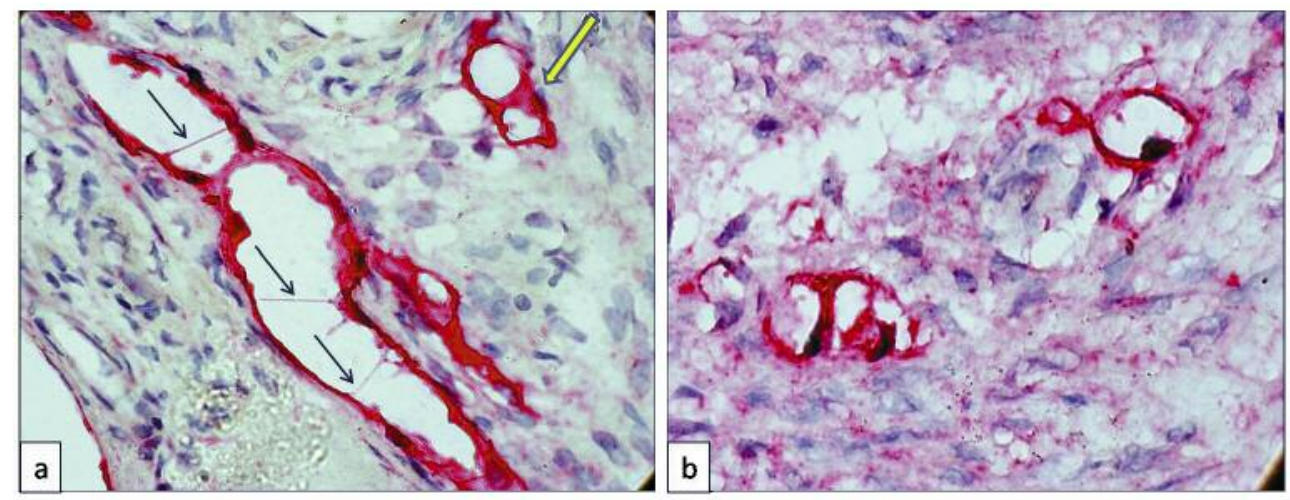

Figure 1. Intussusception often met in peritumoral lymphatic vessels, observed in the initial stage of cytoplasmic extensions (a, blue arrow) or when completely established ( $a$, yellow arrow). The intussusception was associated with intense lymphatic endothelial cell proliferation (b).
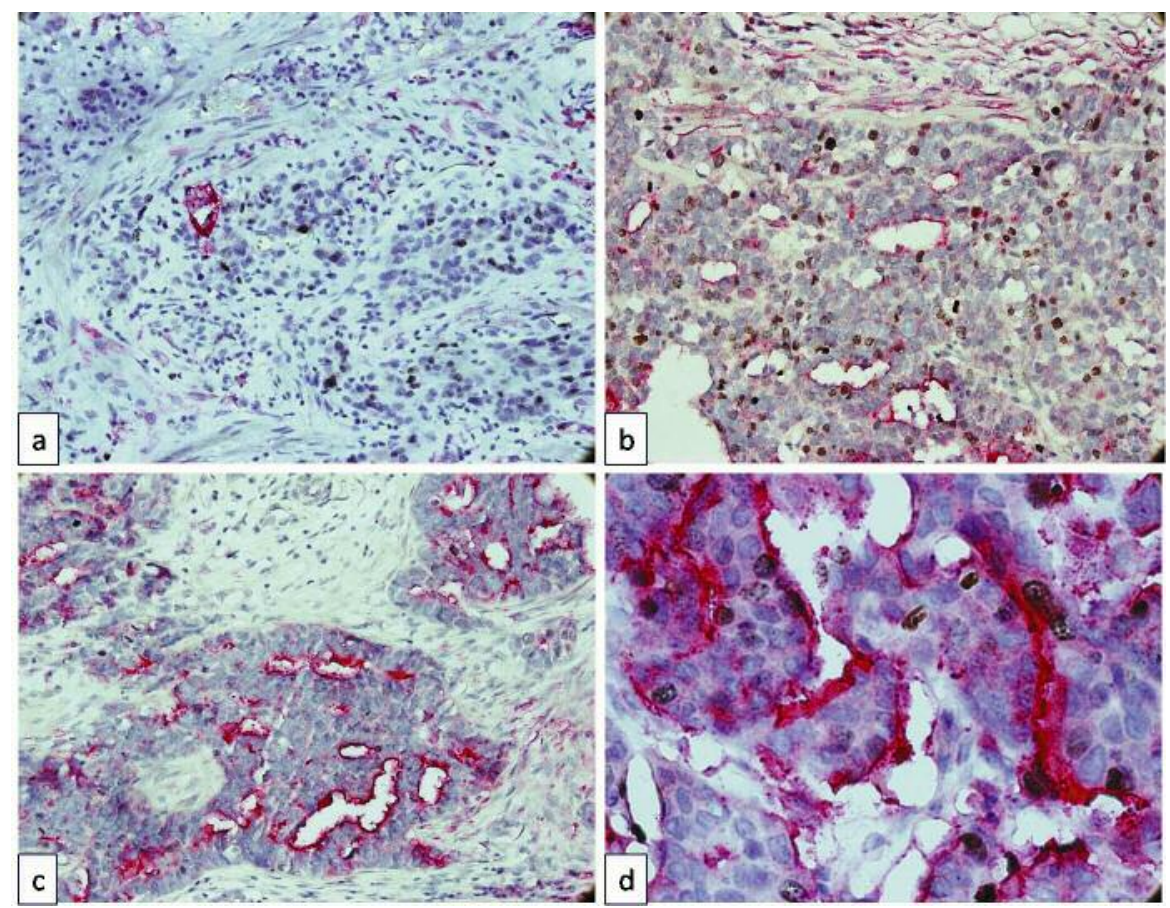

Figure 2. Intratumoral lymphatic vessels in the studied ovarian tumors, distributed as isolated vessels but already with lumen, at the tumor periphery $(a)$, or intratumorally, throughout the entire tumor $(b)$. The lymphatics had continuous walls $(c)$ or were invaded by metastatic tumor cells with destruction of the wall and discontinuity of the D2-40-positive endothelium, with numerous proliferating cells on the circumference of the vessels (d).

In a study conducted by Lin et al. for a period of 18 years, elderly patients diagnosed with ovarian cancer presented the best survival rate following optimal therapy for ovarian cancer (13). The most negative characteristic of ovarian cancer therapy is represented by the early development of resistance to therapy. This reflects the fact that proliferation and metastasis are constant in this type of cancer. Due to these facts, research efforts conducted in this field are attempting to find a marker with an increased specificity and sensitivity that may be used as a targeted therapy in ovarian cancer. Many features of this disease is being intensely studied, from the involvement of epigenetics in the mechanism of therapy resistance (14) to research conducted on cancer stem cells (15, 16). This research adds to that carried out on anti-angiogenic therapy, which targets newly-formed vessels that are intensely studied in ovarian cancer (17-19). 


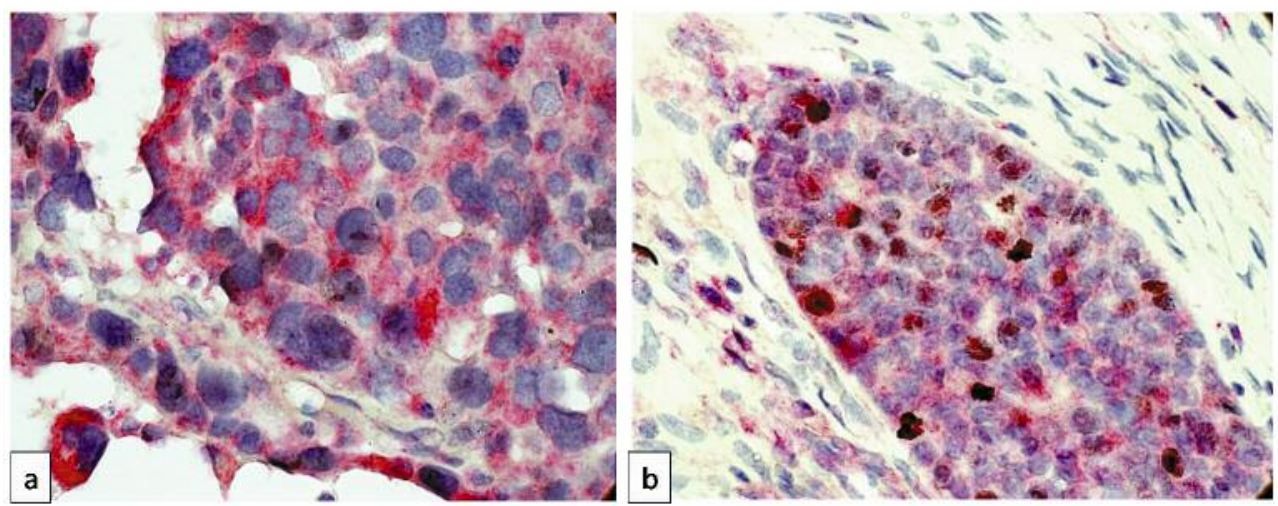

Figure 3. Intense expression in cells with severe nuclear anaplasia (a) and in cells from the proliferative front (b). Note that the invasive cells were not proliferative.

Table I. Analysis of the correlation between the peritumoral (LMVDP) and intratumoral (LMVDT) lymphatic vascular density with the proliferative grade of the lymphatic endothelial cells from the intratumoral and peritumoral vessels.

\begin{tabular}{|c|c|c|c|c|c|c|c|c|}
\hline & & & \multirow[b]{2}{*}{ LMVDT } & \multicolumn{3}{|c|}{ Peritumoral } & \multicolumn{2}{|c|}{ Intratumoral } \\
\hline & & & & LMVDP & $\mathrm{D} 2-40^{+} \mathrm{Ki} 67^{+}$ & D2-40+Ki67- & D2-40+Ki67- & D- $240^{+} \mathrm{Ki}^{6} 7^{+}$ \\
\hline & LMVDT & Pearson correlation & 1 & -0.157 & $0.310^{*}$ & 0.220 & $0.508^{* *}$ & 0.092 \\
\hline & & $p$-Value & & 0.263 & 0.024 & 0.114 & $<0.0001$ & 0.514 \\
\hline & LMVDP & Pearson correlation & -0.157 & 1 & 0.061 & $0.861 * *$ & -0.018 & -0.059 \\
\hline & & $p$-Value & 0.263 & & 0.666 & $<0.0001$ & 0.899 & 0.673 \\
\hline \multirow[t]{4}{*}{ Peritumoral } & $\mathrm{D} 2-40^{+} \mathrm{Ki} 67^{+}$ & Pearson correlation & $0.310 *$ & 0.061 & 1 & 0.129 & 0.006 & 0.057 \\
\hline & & $p$-Value & 0.024 & 0.666 & & 0.356 & 0.968 & 0.685 \\
\hline & D2-40+Ki67- & Pearson correlation & 0.220 & $0.861 * *$ & 0.129 & 1 & -0.012 & -0.072 \\
\hline & & $p$-Value & 0.114 & $<0.0001$ & 0.356 & & 0.934 & 0.610 \\
\hline \multirow[t]{4}{*}{ Intratumoral } & D2-40+Ki67- & Pearson correlation & $0.508 * *$ & -0.018 & 0.006 & -0.012 & 1 & 0.173 \\
\hline & & $p$-Value & $<0.0001$ & 0.899 & 0.968 & 0.934 & & 0.217 \\
\hline & $\mathrm{D} 2-40^{+} \mathrm{Ki} 67^{+}$ & Pearson correlation & 0.092 & -0.059 & 0.057 & -0.072 & 0.173 & 1 \\
\hline & & $p$-Value & 0.514 & 0.673 & 0.685 & 0.610 & 0.217 & \\
\hline
\end{tabular}

*Correlation is significant at the 0.05 level (2-tailed). **Correlation is significant at the 0.01 level (2-tailed). N=53.

Table II. The correlation between the tumor grading $(G)$ and intratumoral lymphatic endothelial proliferation.

\begin{tabular}{llcc}
\hline & & $\mathrm{D} 2-40^{+} / \mathrm{Ki} 67^{+}$, intratumoral & $\mathrm{G}$ \\
\hline $\mathrm{D} 2-40^{+}$ & Pearson correlation & 1 & $0.242^{*}$ \\
$\mathrm{Ki} 67^{+}$intratumoral & $p$-Value & & 0.042 \\
$\mathrm{G}$ & Pearson correlation & $0.242^{*}$ & 1 \\
& $p$-Value & 0.042 & \\
\hline
\end{tabular}

*Correlation is significant at the 0.05 level (1-tailed). $\mathrm{N}=53$.

Paradoxically, although lymphatic vessels seem to possess an important metastatic role in the study of routine histological specimens, they have been less studied in ovarian carcinomas. Search results on PubMed using the key words "ovarian cancer lymphatic vessels" revealed 102 articles. However, using key words "ovarian cancer lymphangiogenesis" for the search results gave only 37 scientific titles. Most published data are limited to the study of the microvessel density (20-22), while very few data are available on angiogenic and lymphangiogenic growth factors $(23,24)$. These factors seem to be almost completely neglected in the process of endothelial proliferation, not only in blood vessels but also in lymphatic vessels. Therefore, our study 
proposed the quantification of tumor lymphangiogenesis in ovarian cancer specimens, the analysis being made in two different zones, the peritumoral and intratumoral areas. Additionally, microvessel density was taken into consideration when estimating the lymphatic endothelial proliferative rate.

In 2010, Yang et al. detected and cultured lymphatic endothelial cells that were associated with epithelial ovarian tumors together with ovarian carcinoma cell lines. They showed that lymphatic endothelial cells from epithelial ovarian tumors have the ability to interact with cancer cells and are able to increase their migrational and invasive characteristics, most probably through a mechanism that is mediated via matrix metalloproteinase 9 (MMP9)/tissue inhibitor of matrix metalloproteinase 2 (TIMP2) activation (25). Based on their results, four articles that focused on the role of lymphangiogenesis in ovarian carcinoma progression, were then published. These scientific studies attempted to find molecular factors that may be used in order to promote that hypothesis. Based on these articles, Zhang et al. conducted studies in which they demonstrated that high-mobility group box 1 protein (HMGB1, amphoterin) and ovarian tumorassociated macrophages facilitate lymphangiogenesis through promoting lymphatic endothelial cell proliferation, migration and capacity to organize as capillary-like structures (26). Our results concur with these findings. We observed an increase of intraluminal pillars mostly in the peritumoral lymphatic vessels and of lymphatic vascular density in the areas where we encountered associations between the lymphatic vessels and the massive D2-40-positive groups of macrophages. Moreover, lymphatic endothelial proliferation was increased, lymphatic endothelial cells that presented intraluminal extended pillars very often exhibited proliferative aspects. Compared to the above described study, our results provide original information due to the fact that we observed morphological and immunohistochemical aspects that could influence future therapeutic decisions in and management of ovarian cancer. Our study differentially quantified LMVDP and LMVDT, significant correlations being observed between LMVDT and the proliferative grade of peritumoral lymphatic cells. This suggests that in advanced stages of ovarian carcinoma, the tumor continues to develop lymphatic vessels.

Podoplanin has little been subject to research studies. Podoplanin is known as a marker of progression and metastasis in various tumor types (1), and its expression in seminomas, epithelioid mesothelioma, and hemagioblastomas is well known (27). Podoplanin is also expressed in adrenal tumors, schwannomas and in tumors that originate from the skin appendages (28).

In ovarian tumors, podoplanin has already been studied as an imunohistochemical diagnostic marker for ovarian tumors derived from germinal cells (29) and also as an epithelio-mesenchymal transition marker in ovarian tumors (30). Kitano and coworkers studied the involvement of podoplanin in the tumor stroma in association with tumor fibroblasts and indirectly with the induction of lymphangiogenesis. They demonstrated its pro-lymphangiogenic role in numerous tumor types, including ovarian tumors (31).

Almeida et al. demonstrated that the LMVDT is higher in primary mucinous ovarian tumors in comparison to secondary types, but this did not represent a differentiation marker of primary from secondary tumors, as was evidenced in the case of keratin 7 and mucin 2 (MUC2) expression (32).

Regarding our observation concerning the existence of lymphatic vessels in the albicans body, we conclude that they could determine the destruction of steroidogenic cells, although this has not yet been fully elucidated (33).

Our study represents the first description of endothelial cell proliferation in ovarian cancer, differentially analyzed in the peritumoral and intratumoral areas, and suggests lymphatic vascular network progression even in advanced stages of ovarian carcinoma. We noticed more than one stage of lymphatic intussusception. In the initial stage, lymphatic endothelial cells present pointed expansions with a very fine structure which resembles pseudopod-like structures encountered in other cell types. Recently, Martin-Villar et al. described the involvement of podoplanin in the induction of these cytoplasmic expansions (34). Most probably, in ovarian tumors, podoplanin possesses the same function, which is not restricted to the level of lymphatic endothelial cells. Its expression was also observed in tumor cells located at the invasion front, which was described in our study as being intensely positive for podoplanin. The involvement of these cell structures in tumor invasion was evidenced in other tumor types such as breast tumors, melanomas and gliomas (35). All these data support the need to study podoplanin as a possible maker of invasion and metastasis in ovarian tumors. This could be useful for its characterization as a possible anti-metastatic therapeutic target on one hand, and its use in combination with anti-lymphangiogenic therapeutic agents on the other hand. Targeted therapies are already being applied in other tumor types, such as breast tumors. Through its differential expression, either cytoplasmic or apically restricted in ovarian tumors, our data suggest that tumor cells are able to secrete podoplanin and to release it into the tumor microenvironment.

\section{Acknowledgements}

The Authors are grateful to Dr. Flavia Baderca for her technical support in providing paraffin embedded specimens. This work was supported by Victor Babes University of Medicine and Pharmacy Timisoara, Romania.

\section{References}

1 Raica M, Cimpean AM and Ribatti D: The role of podoplanin in tumor progression and metastasis. Anticancer Res 28: 29973006, 2008. 
2 Herzog BH, Fu J, Wilson SJ, Hess PR, Sen A, McDaniel JM, Pan Y, Sheng M, Yago T, Silasi-Mansat R, McGee S, May F, Nieswandt B, Morris AJ, Lupu F, Coughlin SR, McEver RP, Chen H, Kahn ML and Xia L: Podoplanin maintains high endothelial venule integrity by interacting with platelet CLEC2. Nature 502: 105-109, 2013.

3 Pan Y and Xia L: Emerging roles of podoplanin in vascular development and homeostasis. Front Med 9: 421-430, 2015.

4 Cîrligeriu L, Cimpean AM, Raica M and Doroş CI: Dual role of podoplanin in oral cancer development. In Vivo 28: 341-347, 2014.

5 Cimpean AM, Mazuru V, Cernii A, Ceausu R, Saptefrati L, Cebanu A, Fit AM and Raica M: Detection of early lymphangiogenesis by lymphatic microvascular density and endothelial proliferation status in preneoplastic and neoplastic lesions of the uterine cervix. Pathol Int 61: 395-400, 2011.

6 Saptefraţi L, Cîmpean AM, Ciornîi A, Ceauşu R, Eşanu N and Raica M: Identification of lymphatic vessels and prognostic value of lymphatic microvessel density in lesions of the uterine cervix. Rom J Morphol Embryol 50: 589-594, 2009.

7 Cheng Y, Wang K, Ma W, Zhang X, Song Y, Wang J, Wang N, Song Q, Cao F, Tan B and Yu J: Cancer-associated fibroblasts are associated with poor prognosis in esophageal squamous cell carcinoma after surgery. Int J Clin Exp Med 8: 1896-1903, 2015.

8 Kato Y and Kaneko MK: A cancer-specific monoclonal antibody recognizes the aberrantly glycosylated podoplanin. Sci Rep 4: 5924, 2014.

9 Kleppe M, Kraima AC, Kruitwagen RF, Van Gorp T, Smit NN, van Munsteren JC and DeRuiter MC: Understanding lymphatic drainage pathways of the ovaries to predict sites for sentinel nodes in ovarian cancer. Int J Gynecol Cancer 25: 1405-1414, 2015.

10 Almeida BG, Bacchi CE, Carvalho JP, Ferreira CR and Carvalho FM: The role of intratumoral lymphovascular density in distinguishing primary from secondary mucinousovarian tumors. Clinics (Sao Paulo) 69: 660-665, 2014

11 Oe S, Hasegawa K, Nagase S, Kato R, Torii Y and Udagawa Y: Expression of podoplanin in epithelial ovarian carcinomas and its potential as a marker for clear cell adenocarcinoma. Int $\mathrm{J}$ Gynecol Pathol 29: 405-410, 2010.

12 Kitano H, Kageyama S, Hewitt SM, Hayashi R, Doki Y, Ozaki Y, Fujino S, Takikita M, Kubo H and Fukuoka J: Podoplanin expression in cancerous stroma induces lymphangiogenesis and predicts lymphatic spread and patient survival. Arch Pathol Lab Med 134: 1520-1527, 2010.

13 Lin JJ, Egorova N, Franco R, Prasad-Hayes M and Bickell NA: Ovarian cancer treatment and survival trends among women older than 65 years of age in the United States, 1995-2008. Obstet Gynecol 127: 81-89, 2016.

14 Nogales V, Reinhold WC, Varma S, Martinez-Cardus A, Moutinho C, Moran S, Heyn H, Sebio A, Barnadas A, Pommier $\mathrm{Y}$ and Esteller $\mathrm{M}$ : Epigenetic inactivation of the putative DNA/RNA helicase SLFN11 in human cancer confers resistance to platinum drugs. Oncotarget 7: 3084-3097, 2016.

15 Virant-Klun I, Ståhlberg A, Kubista $M$ and Skutella T: MicroRNAs: From female fertility, germ cells, and stem cells to cancer in humans. Stem Cells Int 2016: 3984937, 2016.

16 Laganà AS, Colonese F, Colonese E, Sofo V, Salmeri FM, Granese R, Chiofalo B, Ciancimino L and Triolo O: Cytogenetic analysis of epithelial ovarian cancers stem cells: an overview on new diagnostic and therapeutic perspectives. Eur J Gynaecol Oncol 36: 495-505, 2015.
17 Duckworth C, Zhang L, Carroll SL, Ethier SP and Cheung HW: Overexpression of GAB2 in ovarian cancer cells promotes tumor growth and angiogenesis by upregulating chemokine expression. Oncogene doi: 10.1038/onc.2015.472, 2015.

$18 \mathrm{Li} \mathrm{X}$, Zhu S, Hong C and Cai H: Angiogenesis inhibitors for patients with ovarian cancer: A meta-analysis of 12 randomized controlled trials. Curr Med Res Opin 11: 1-21, 2015.

19 Previs RA, Armaiz-Pena GN, Lin YG, Davis AN, Pradeep S, Dalton HJ, Hansen JM, Merritt WM, Nick AM, Langley RR, Coleman RL and Sood AK: Dual metronomic chemotherapy with nab-paclitaxel and topotecan has potent antiangiogenic activity in ovarian cancer. Mol Cancer Ther 14: 2677-2686, 2015.

20 Pansrikaew P, Cheewakriangkrai C, Taweevisit M, Khunamornpong S and Siriaunkgul S: Correlation of mast cell density, tumor angiogenesis, and clinical outcomes in patients with endometrioid endometrial cancer. Asian Pac J Cancer Prev 11: 623-626, 2010.

21 Kwak Y, Lee HE, Kim WH, Kim DW, Kang SB and Lee HS: The clinical implication of cancer-associated microvasculature and fibroblast in advanced colorectalcancer patients with synchronous or metachronous metastases. PLoS One 9: e91811, 2014.

22 Schoppmann SF, Horvat R and Birner P: Lymphatic vessels and lymphangiogenesis in female cancer: mechanisms, clinical impact and possible implications for anti-lymphangiogenic therapies (Review). Oncol Rep 9: 455-460, 2002.

23 Mahner S, Woelber L, Mueller V, Witzel I, Prieske K, Grimm D, Keller-V Amsberg G and Trillsch F: Beyond bevacizumab: an outlook to new anti-angiogenics for the treatment of ovarian cancer. Front Oncol 5: 211, 2015.

24 Ranjbar R, Nejatollahi F, Nedaei Ahmadi AS, Hafezi H and Safaie A: Expression of vascular endothelial growth factor (VEGF) and epidermal growth factor receptor (EGFR) in patients with serous ovarian carcinoma and their clinical significance. Iran J Cancer Prev 8: e3428, 2015.

25 Yang S, Zhu X, Cai L, Cheng H, Zhao R, Wang H, Zhao H and Wang Z: Role of tumor-associated lymphatic endothelial cells in metastasis: a study of epithelial ovarian tumor in vitro. Cancer Sci 101: 679-685, 2010

26 Zhang W, Tian J and Hao Q: HMGB1 combining with tumorassociated macrophages enhanced lymphangiogenesis in human epithelial ovarian cancer. Tumour Biol 35: 2175-2186, 2014.

27 Ordóñez NG: Podoplanin: a novel diagnostic immunohistochemical marker. Adv Anat Pathol 13: 83-88, 2006.

28 Kalof AN and Cooper K: D2-40 immunohistochemistry--so far! Adv Anat Pathol 16: 62-64, 2009.

29 Chang MC, Vargas SO, Hornick JL, Hirsch MS, Crum CP and Nucci MR: Embryonic stem cell transcription factors and D2-40 (podoplanin) as diagnostic immunohistochemical markers in ovarian germ cell tumors. Int J Gynecol Pathol 28: 347-355, 2009.

30 Okamoto S, Okamoto A, Nikaido T, Saito M, Takao M, Yanaihara N, Takakura S, Ochiai K and Tanaka T: Mesenchymal to epithelial transition in the human ovarian surface epithelium focusing on inclusion cysts. Oncol Rep 21: 1209-1214, 2009.

31 Kitano H, Kageyama S, Hewitt SM, Hayashi R, Doki Y, Ozaki Y, Fujino S, Takikita M, Kubo H and Fukuoka J: Podoplanin expression in cancerous stroma induces lymphangiogenesis and predicts lymphatic spread and patient survival. Arch Pathol Lab Med 134: 1520-1527, 2010. 
32 Almeida BG, Bacchi CE, Carvalho JP, Ferreira CR and Carvalho FM: The role of intratumoral lymphovascular density in distinguishing primary from secondary mucinousovarian tumors. Clinics 69: 660-665, 2014.

33 Abe H, Al-zi'abi MO, Sekizawa F, Acosta TJ, Skarzynski DJ and Okuda K: Lymphatic involvement in the disappearance of steroidogenic cells from the corpus luteum during luteolysis. PLoS One 9: e88953, 2014.

34 Martín-Villar E, Borda-d'Agua B, Carrasco-Ramirez P, Renart J, Parsons M, Quintanilla M and Jones GE: Podoplanin mediates
ECM degradation by squamous carcinoma cells through control of invadopodia stability. Oncogene 34: 4531-4544, 2015.

35 Stylli SS, Kaye AH and Lock P: Invadopodia: at the cutting edge of tumour invasion. J Clin Neurosci 15: 725-737, 2008.

Received July 21, 2016

Revised September 2, 2016

Accepted September 20, 2016 